\title{
Transformation à la Foata for Special Kinds of Descents and Excedances
}

\author{
Jean-Luc Baril and Sergey Kirgizov \\ LIB, Université Bourgogne Franche-Comté, B.P. 47 870, 21078 Dijon-Cedex, France \\ Email: barjl@u-bourgogne.fr, sergey.kirgizov@u-bourgogne.fr
}

Received: January 6, 2021, Accepted: March 17, 2021, Published: March 26, 2021

The authors: Released under the CC BY-ND license (International 4.0)

ABstract: A pure excedance in a permutation $\pi=\pi_{1} \pi_{2} \ldots \pi_{n}$ is a position $i<\pi_{i}$ such that there is no $j<i$ with $i \leq \pi_{j}<\pi_{i}$. We present a one-to-one correspondence on the symmetric group that transports pure excedances to descents of a special kind. As a byproduct, we prove that the popularity of pure excedances equals those of pure descents on permutations, while their distributions are different.

Keywords: Cycle; Descent; Distribution; Excedance; Permutation; Popularity; Statistic 2020 Mathematics Subject Classification: 05A05; 05A15; 05A19

\section{Introduction and notations}

The distribution of the number of descents has been widely studied on several classes of combinatorial objects such as permutations [14], cycles [7,8], and words [3, 10]. Many interpretations of this statistic appear in several fields as Coxeter groups [4,11] or lattice path theory [12]. One of the most famous result involves the Foata fundamental transformation [9] to establish a one-to-one correspondence between descents and excedances on permutations. This bijection provides a more straightforward proof than those of MacMahon [14] for the equidistribution of these two Eulerian statistics.

In this paper, we present a bijection à la Foata on the symmetric group that exchanges pure excedances with special kind of descents defined as a mesh pattern $p_{2}[6]$ (see below for the definition of this pattern). Then, we deduce that the popularities (but not the distributions) of pure descents [2] and pure excedances are the same. This common popularity is given by the generalized Stirling number $n ! \cdot\left(H_{n}-1\right)$ (see Sequence A001705 in [15]) where $H_{n}=\sum_{k=1}^{n} \frac{1}{k}$ is the $n$th harmonic number. Finally, we conjecture the existence of a bijection on the symmetric group that exchanges pure excedances and $p_{2}$ while preserving the number of cycles.

Let $S_{n}$ be the set of permutations of length $n$, i.e., all bijections from $[n]=\{1,2, \ldots, n\}$ into itself. The one-line representation of a permutation $\pi \in S_{n}$ is $\pi=\pi_{1} \pi_{2} \ldots \pi_{n}$ where $\pi_{i}=\pi(i), 1 \leq i \leq n$. For $\sigma \in S_{n}$, the product $\sigma \cdot \pi$ is the permutation $\sigma\left(\pi_{1}\right) \sigma\left(\pi_{2}\right) \ldots \sigma\left(\pi_{n}\right)$. A $\ell$-cycle $\pi=\left\langle i_{1}, i_{2}, \ldots, i_{\ell}\right\rangle$ in $S_{n}$ is a $n$-length permutation satisfying $\pi\left(i_{1}\right)=i_{2}, \pi\left(i_{2}\right)=i_{3}, \ldots, \pi\left(i_{\ell-1}\right)=i_{\ell}, \pi\left(i_{\ell}\right)=i_{1}$ and $\pi(j)=j$ for $j \in[n] \backslash\left\{i_{1}, i_{2}, \ldots, i_{\ell}\right\}$. For $1 \leq k \leq n$, we denote by $C_{n, k}$ the set of all $n$-length permutations admitting a decomposition in a product of $k$ disjoint cycles. The set $C_{n, k}$ is counted by the signless Stirling numbers of the first kind $c(n, k)$ defined by

$$
c(n, k)=(n-1) c(n-1, k)+c(n-1, k-1)
$$

where $c(n, k)=0$ if $n=0$ or $k=0$, except $c(0,0)=1$ (see [16,17] and Sequence A132393 in [15]). These numbers also enumerate $n$-length permutations $\pi$ having $k$ left-to-right maxima, i.e., positions $i \in[n]$ such that $\pi_{j}<\pi_{i}$ for $j<i$ (see [16]), and permutations $\pi \in S_{n}$ with $k-1$ pure descents, i.e., descents $\pi_{i}>\pi_{i+1}$ where there is no $j<i$ such that $\pi_{j} \in\left[\pi_{i+1}, \pi_{i}\right]$ (see [2]). Note that a pure descent can be viewed as an occurrence of the mesh pattern $\left(21, L_{1}\right)$ where $L_{1}=\{1\} \times[0,2] \cup\{(0,1)\}$. Indeed, for a $k$-length permutation $\sigma$ and a subset $R \subseteq[0, k] \times[0, k]$, an occurrence of the mesh pattern $(\sigma, R)$ in a permutation $\pi$ is an occurrence of $\sigma$ in $\pi$ with the additional restriction that no element of $\pi$ lies inside the shaded regions defined by $R$, where $(i, j) \in R$ means the square having bottom left corner $(i, j)$ in the graphical representation $\left\{\left(i, \sigma_{i}\right), i \in[k]\right\}$ of $\sigma$. For instance, an occurrence of the mesh pattern $p_{1}$ in Figure 1 corresponds to an occurrence of a pure descent. See [6] for a more detailed definition of mesh patterns.

Regarding this interpretation of pure descents in terms of mesh patterns, we define other kinds of descents by the mesh patterns $p_{i}=\left(21, L_{i}\right), p_{i}^{\prime}=\left(21, R_{i}\right)$ with $L_{i}=\{1\} \times[0,2] \cup\{(0, i)\}$ and $R_{i}=\{1\} \times[0,2] \cup\{(2, i)\}$ for $0 \leq i \leq 2$. Modulo the trivial symmetries on permutations (reverse and complement), it is straightforward to see that $p_{0}, p_{1}$ and $p_{2}$ are respectively in the same distribution class as $p_{2}^{\prime}, p_{1}^{\prime}$ and $p_{0}^{\prime}$. Then, we deal with only mesh 
patterns $p_{i}, i \in[0,2]$. We refer to Figure 1 for a graphical illustration. On the other hand, we define a pure excedance as an occurrence of an excedance, i.e. $\pi_{i}>i$, with the additional restriction that there is no point $\left(j, \pi_{j}\right)$ such that $1 \leq j \leq i-1$ with $i \leq \pi_{j}<\pi_{i}$. Although such a pattern (called pex) is not a mesh pattern, we can represent it graphically as shown in Figure 1.

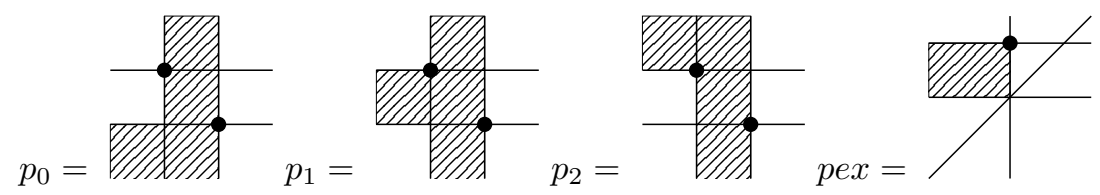

Figure 1: Illustration of the mesh patterns $p_{0}, p_{1}, p_{2}$ and $p e x ; p_{1}$ and pex correspond respectively to a pure descent and a pure excedance.

A statistic is an integer-valued function from a set $\mathcal{A}$ of $n$-length permutations (we use the boldface to denote statistics). For a pattern $p$, we define the pattern statistic $\mathbf{p}: \mathcal{A} \rightarrow \mathbb{N}$ where the image $\mathbf{p} \pi$ of $\pi \in \mathcal{A}$ by $\mathbf{p}$ is the number of occurrences of $p$ in $\pi$. The popularity of $p$ in $\mathcal{A}$ is the total number of occurrences of $p$ over all objects of $\mathcal{A}$, that is $\sum_{a \in \mathcal{A}} \mathbf{p} a$ (see [5] for instance). Below, we present statistics that we use throughout the paper:

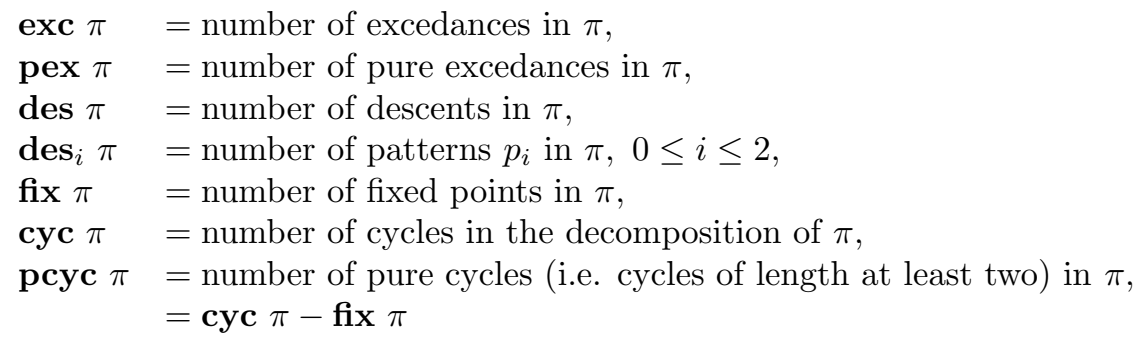

We organize the paper as follows. In Section 2, we focus on patterns $p_{i}, 0 \leq i \leq 2$. We prove that the statistics $\mathbf{d e s}_{0}$ and $\mathbf{d e s}_{1}$ are equidistributed by giving algebraic and bijective proofs. Next, we provide the bivariate exponential generating function for the distribution of $p_{2}$, and we deduce that $p_{2}$ has the same popularity as $p_{0}$ and $p_{1}$, without having the same distribution. In Section 3, we present a bijection on $S_{n}$ that transports pure excedances into patterns $p_{2}$. Notice that the Foata's first transformation [9] is not a candidate for such a bijection. As a consequence, pure descents and pure excedances are equipopular on $S_{n}$, but they do not have the same distribution. Combining all these results, we deduce that patterns $p_{i}, 0 \leq i \leq 2$, and pex are equipopular on the symmetric group $S_{n}$. Finally we present two conjectures about the equidistribution of $\left(\mathbf{c y c}_{\mathbf{c}} \mathbf{d e s}_{2}\right)$ and (cyc, pex), and that of (des, $\left.\mathbf{d e s}_{2}\right)$ and (exc, pex).

\section{The statistics $\operatorname{des}_{i}, 0 \leq i \leq 2$}

For $0 \leq i \leq 2$, let $A_{n, k}^{i}$ be the set of $n$-length permutations having $k$ occurrences of $p_{i}$, and denote by $a_{n, k}^{i}$ its cardinality. Let $A^{i}(x, y)$ be the bivariate exponential generating function $\sum_{n=0}^{\infty} \sum_{k=0}^{n-1} a_{n, k}^{i} \frac{x^{n}}{n !} y^{k}$. In [2, 13], it is proved that $a_{n, k}^{1}$ equals the signless Stirling numbers of the first kind $c(n, k+1)$ (see Sequence A132393 in [15]). Indeed, a permutation $\sigma \in A_{n, k}^{1}$ can be uniquely obtained from an $(n-1)$-length permutation $\pi$ by one of the two following constructions:

(i) if $\pi \in A_{n-1, k-1}^{1}$, then we increase by one all values of $\pi$ greater than or equal to $\pi_{n-1}$, and we add $\pi_{n-1}$ at the end;

(ii) if $\pi \in A_{n-1, k}^{1}$, then we increase by one all values of $\pi$ greater than or equal to a given value $x \leq n, x \neq \pi_{n-1}$ and we add $x$ at the end.

Then, we deduce the recurrence relation $a_{n, k}^{1}=a_{n-1, k-1}^{1}+(n-1) a_{n-1, k}^{1}$ with $a_{n, 0}^{1}=(n-1)$ ! for $n \geq 1$, $a_{0,0}^{1}=1$ and the bivariate exponential generating function is

$$
A^{1}(x, y)=\frac{1}{y(1-x)^{y}}-\frac{1}{y}+1
$$

which proves that $a_{n, k}^{1}=c(n, k+1)$.

Below, we prove that $a_{n, k}^{1}$ also counts $n$-length permutations having $k$ occurrences of the pattern $p_{0}$. 
Theorem 2.1. The number $a_{n, k}^{0}$ of n-length permutations having $k$ occurrences of pattern $p_{0}$ equals $a_{n, k}^{1}=$ $c(n, k+1)$.

Proof. An $n$-length permutation $\sigma \in A_{n, k}^{0}$ can be uniquely obtained from an $(n-1)$-length permutation $\pi$ by one of the two following constructions:

(i) if $\pi \in A_{n-1, k-1}^{0}$, then we increase by one all values of $\pi$ and we add 1 at the end;

(ii) if $\pi \in A_{n-1, k}^{0}$, then we increase by one all values of $\pi$ greater than or equal to a given value $x, 1<x \leq n$, and we add $x$ at the end.

We deduce the recurrence relation $a_{n, k}^{0}=a_{n-1, k-1}^{0}+(n-1) a_{n-1, k}^{0}$ with the initial condition $a_{n, 0}^{0}=(n-1)$ !, and then $a_{n, k}^{0}=a_{n, k}^{1}=c(n, k+1)$.

Now, we focus on the distribution of the pattern $p_{2}$. Table 1 provides exact values for small sizes.

Theorem 2.2. We have

$$
A^{2}(x, y)=\frac{e^{x(1-y)}}{(1-x)^{y}}
$$

and the general term $a_{n, k}^{2}$ satisfies for $n \geq 2$ and $1 \leq k \leq\left\lfloor\frac{n}{2}\right\rfloor$

$$
a_{n, k}^{2}=n a_{n-1, k}^{2}+(n-1) a_{n-2, k-1}^{2}-(n-1) a_{n-2, k}^{2}
$$

with the initial conditions $a_{n, 0}^{2}=1$ and $a_{n, k}^{2}=0$ for $n \geq 0$ and $k>\left\lfloor\frac{n}{2}\right\rfloor$ (see Table 1 and Sequence A136394 in [15]).

Proof. Let $\sigma=\sigma_{1} \sigma_{2} \ldots \sigma_{n}$ denote a permutation of length $n$ having $k$ occurrences of pattern $p_{2}$. Let $u_{n, k}$ (resp. $v_{n, k}$ ) be the number of such permutations satisfying $\sigma_{n}=n$ (resp. $\sigma_{n}<n$ ). Obviously, we have

$$
a_{n, k}^{2}=u_{n, k}+v_{n, k} .
$$

A permutation $\sigma$ with $\sigma_{n}=n$ can be uniquely constructed from an $(n-1)$-length permutation $\pi$ as $\sigma=$ $\pi_{1} \pi_{2} \ldots \pi_{n-1} n$. No new occurrences of $p_{2}$ are created, and we obtain

$$
u_{n, k}=a_{n-1, k}^{2} .
$$

A permutation $\sigma$ satisfying $\sigma_{n}<n$ can be uniquely obtained from an $(n-1)$-length permutation $\pi$ by adding a value $x<n$ on the right side of its one-line notation, after increasing by one all the values greater than or equal to $x$. This construction creates a new pattern $p_{2}$ if and only if $\pi$ ends with $n-1$. Thus, we deduce

$$
v_{n, k}=(n-1) u_{n-1, k-1}+(n-1) v_{n-1, k} .
$$

Combining the equations, we obtain for $n \geq 2$ and $k \geq 1$

$$
a_{n, k}^{2}=n a_{n-1, k}^{2}+(n-1) a_{n-2, k-1}^{2}-(n-1) a_{n-2, k}^{2},
$$

which implies the following differential equation

$$
\frac{\partial A^{2}(x, y)}{\partial x}=(y-1) x A^{2}(x, y)+\frac{\partial\left(x A^{2}(x, y)\right)}{\partial x}, \text { where } A^{2}(x, 0)=1 .
$$

A simple calculation provides the claimed closed form for the generating function $A^{2}(x, y)$.

Corollary 2.1. For $0 \leq i \leq 2$, the patterns $p_{i}$ are equipopular on $S_{n}$. Their popularity is given by the generalized Stirling number $n ! \cdot\left(H_{n}-1\right)$ (see Sequence A001705 in [15]) where $H_{n}=\sum_{k=1}^{n} \frac{1}{k}$ is the nth harmonic number.

Proof. The generating function of the popularity is directly deduced from the bivariate generating function of pattern distribution by calculating

$$
\left.\frac{\partial A^{1}(x, y)}{\partial y}\right|_{y=1}=\left.\frac{\partial A^{2}(x, y)}{\partial y}\right|_{y=1} .
$$

The statistic $\mathbf{d e s}_{2}$ has a different distribution from $\mathbf{d e s}_{0}$ and $\mathbf{d e s}_{1}$, but the three patterns $p_{0}, p_{1}, p_{2}$ have the same popularity. Below we present a bijection on $S_{n}$ that transports the statistic des $_{2}$ to the statistics pcyc $=$ cyc - fix. 


\begin{tabular}{c|cccccccc}
$k \backslash n$ & 1 & 2 & 3 & 4 & 5 & 6 & 7 & 8 \\
\hline 0 & 1 & 1 & 1 & 1 & 1 & 1 & 1 & 1 \\
1 & & 1 & 5 & 20 & 84 & 409 & 2365 & 16064 \\
2 & & & & 3 & 35 & 295 & 2359 & 19670 \\
3 & & & & & & 15 & 315 & 4480 \\
4 & & & & & & & & 105 \\
$\ldots$ & & & & & & & & $\ldots$ \\
\hline$\sum$ & 1 & 2 & 6 & 24 & 120 & 720 & 5040 & 40320
\end{tabular}

Table 1: Number of $n$-length permutations having $k$ occurrences of $p_{2}$ for $0 \leq k \leq 4$ and $1 \leq n \leq 8$.

Theorem 2.3. There is a one-to-one correspondence $\phi$ on $S_{n}$ such that for any $\pi \in S_{n}$, we have

$$
\operatorname{des}_{2} \pi=\operatorname{pcyc} \phi(\pi) \text {. }
$$

Proof. Let $\pi$ be a permutation of length $n$ having $k$ occurrences of $p_{2}$. We decompose

$$
\pi=B_{0} \pi_{i_{1}} A_{1} B_{1} \pi_{i_{2}} A_{2} B_{2} \pi_{i_{3}} \ldots \pi_{i_{k}} A_{k} B_{k}
$$

where

- $\pi_{i_{1}}<\pi_{i_{2}}<\ldots<\pi_{i_{k}}$ are the tops of the occurrences of $p_{2}$, i.e. values $\pi_{i_{j}}>\pi_{i_{j}+1}$ such that there does not exist $\ell<i_{j}$ such that $\pi_{\ell}>\pi_{i_{j}}$,

- $A_{j}$ is a maximal sequence such that all its values are lower than $\pi_{i_{j}}$,

- for $0 \leq j \leq k, B_{j}$ is an increasing sequence such that $\pi_{i_{j}}<\min B_{j}$ and $\max B_{j}<\pi_{i_{j+1}}$.

Now we construct an $n$-length permutation $\phi(\pi)$ with $k$ pure cycles as follows:

$$
\phi(\pi)=\left\langle\pi_{i_{1}} A_{1}\right\rangle \cdot\left\langle\pi_{i_{2}} A_{2}\right\rangle \cdots\left\langle\pi_{i_{k}} A_{k}\right\rangle .
$$

For instance, if $\pi=125346879$ then $\phi(\pi)=\langle 5,3,4\rangle \cdot\langle 8,7\rangle$. The map $\phi$ is clearly a bijection on $S_{n}$ such that $\operatorname{des}_{2} \pi$ equals the number of pure cycles in $\phi(\pi)$.

Note that $\phi^{-1}$ is closely related to the Foata fundamental transformation [9].

\section{The statistic pex of pure excedances}

In order to prove the equidistribution of pex and $\mathbf{d e s}_{2}$, regarding Theorem 2.3, it suffices to construct a bijection on $S_{n}$ that transports pure excedances to pure cycles. Here, we first exhibit a bijection on the set $D_{n}$ of $n$-length derangements (permutations without fixed points), then we extend it to the set of all permutations $S_{n}$.

Any permutation $\pi \in S_{n}$ is uniquely decomposed as a product of transpositions of the following form:

$$
\pi=\left\langle t_{1}, 1\right\rangle \cdot\left\langle t_{2}, 2\right\rangle \cdots\left\langle t_{n}, n\right\rangle
$$

where $t_{i}$ are integers such that $1 \leq t_{i} \leq i$. The transposition array of $\pi$ is defined by $T(\pi)=t_{1} t_{2} \ldots t_{n}$, which induces a bijection $\pi \longmapsto T(\pi)$ from $S_{n}$ to the product set $T_{n}=[1] \times[2] \times \cdots \times[n]$. By Lemma 1 from [1], the number of cycles of a permutation $\pi$ is given by the number of fixed points in $T(\pi)$. Moreover, it is straightforward to check the two following properties:

- if $t_{i}=i$, then $\pi_{i}=i$ if and only if there is no number $j>i$ such that $t_{j}=t_{i}=i$

- if $t_{i}=i$ and $\pi_{i} \neq i$, then $i$ is the minimal element of a cycle of length at least two in $\pi$.

So, we deduce the following lemma.

Lemma 3.1. The transposition array $t_{1} t_{2} \ldots t_{n} \in T_{n}$ corresponds to a derangement if and only if: $t_{i}=i \Rightarrow$ there is a $j>i$ such that $t_{j}=i$.

Given a derangement $\pi=\pi_{1} \pi_{2} \ldots \pi_{n} \in D_{n}$ and its graphical representation $\left\{\left(i, \pi_{i}\right), i \in[n]\right\}$. We say that the square $(i, j) \in[n] \times[n]$ is free if all following conditions hold:

(i) Neither $\pi_{i}$ nor $i$ is a position of a pure excedance;

(ii) $(i, j)$ is not on the first diagonal, i.e. $j \neq i$;

(iii) there does not exist $k>i$ such that $\pi_{k}=j$; 
(iv) $j$ is not a pure excedance such that $j<i$ and $\pi^{-1}(j)<i$;

(v) there does not exist $k<i$, with $\pi_{k}=j>i$ such that all values of the interval $[i, j-1]$ appear on the right of $\pi_{i}$ in $\pi$.

Whenever at least one of the statements above is not satisfied, we say that the square $(i, j)$ is unfree. Notice that if $i$ and $\pi_{i}$ are not the positions of a pure excedance, then the square $\left(i, \pi_{i}\right)$ is always free. So, for a column $i$ of the graphical representation of $\pi$ such that $i$ and $\pi_{i}$ are not the positions of a pure excedance, we label by $j$ the $j$ th free square from the bottom to the top. We refer to Figure 2 for an example of this labeling.

Now we define the map $\lambda$ from $D_{n}$ to the set $T_{n}^{\bullet}$ of transposition arrays of length $n$ satisfying the property of Lemma 3.1.

For a permutation $\pi=\pi_{1} \pi_{2} \ldots \pi_{n} \in D_{n}$, we label its graphical representation as defined above, and $\lambda(\pi)=\lambda_{1} \lambda_{2} \ldots \lambda_{n}$ is obtained as follows:

- if $i$ is a pure excedance in $\pi$, then we set $\lambda_{i}=i$ and $\lambda_{\pi^{-1}(i)}=i$;

- otherwise, $\lambda_{i}$ is the sum of the label of the free square $\left(i, \pi_{i}\right)$ with the number of pure excedances $k<i$ such that $\pi^{-1}(k)<i$.

For instance, if $\pi=681254732111910$ then we obtain $\lambda(\pi)=1124421191910$ (see Figure 2).

Let us consider $i, 1 \leq i \leq n$. If $i$ is a pure excedance of $\pi$, then we fix $\lambda_{i}=i$ and $\lambda_{\pi^{-1}(i)}=i<\pi^{-1}(i)$. Otherwise, the square $(i, i)$ is unfree, and all squares $\left(i, \pi_{k}\right), i+1 \leq k \leq n$, are unfree, which implies that the number of free squares in the $i$ th column is less than or equal to $i$. This means that $\lambda(\pi)$ lies in $T_{n}$. Note that, by construction, all labeled squares do not correspond to any pure excedance. Now let us prove that the square $\left(i, \pi_{i}\right)$ cannot be labeled $i$. Indeed, if $\pi_{i}<i$ then the label of $\left(i, \pi_{i}\right)$ is necessarily at most $\pi_{i} \leq i-1$; otherwise, if $\pi_{i}>i$ then the fact that $i$ is not a pure excedance implies that there is $\pi_{j} \in\left[i, \pi_{i}-1\right]$ with $j<i$. Let us choose the lowest $j$ with this property. Using $(\mathrm{v})$, the square $(i, j)$ is unfree, which implies that the label of $\left(i, \pi_{i}\right)$ is less than or equal to $n$ minus the minimal number of unfree squares $(i, j)$ in column $i$, that is $n-(n-i+1)=i-1$. Moreover, the transposition array $\lambda(\pi)$ has exactly pex $\pi$ fixed points, and for any fixed point $i$ there necessarily exists $j=\pi^{-1}(i)>i$ such that $\lambda_{j}=\lambda_{i}=i$. This implies that $\lambda(\pi) \in T_{n}^{\bullet}$.
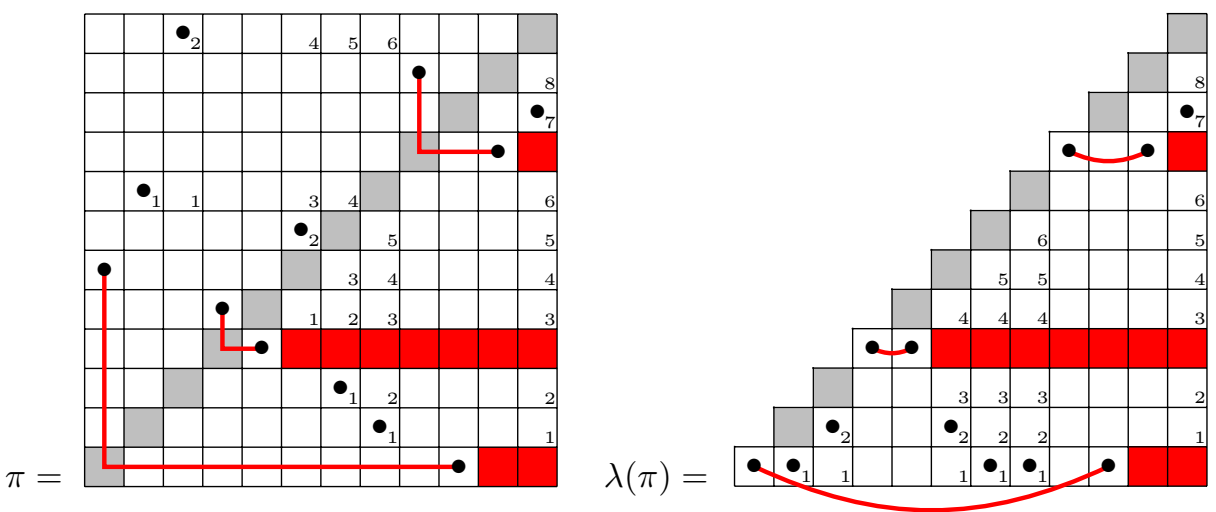

Figure 2: Illustration of the bijection $\lambda$ for $\pi=681254732111910$ and $\lambda(\pi)=1124421191910$.

Theorem 3.1. The map $\lambda$ from $D_{n}$ to $T_{n}^{\bullet}$ is a bijection such that

$$
\operatorname{pex} \pi=\operatorname{fix} \lambda(\pi) \text {. }
$$

Proof. Since the cardinality of $T_{n}^{\bullet}$ equals that of $D_{n}$, and the image of $D_{n}$ by $\lambda$ is contained in $T_{n}^{\bullet}$, it suffices to prove the injectivity.

Let $\pi$ and $\sigma, \pi \neq \sigma$, be two derangements in $D_{n}$. If $\pi$ and $\sigma$ do not have the same pure excedances, then, by construction, $\lambda(\pi)$ and $\lambda(\sigma)$ do not have the same fixed points, and thus $\lambda(\pi) \neq \lambda(\sigma)$.

Now, let us assume that $\pi$ and $\sigma$ have the same pure excedances. If there is a pure excedance $i$ such that $\pi^{-1}(i) \neq \sigma^{-1}(i)$ then the definition implies $\lambda(\pi) \neq \lambda(\sigma)$. Otherwise the two permutations have the same pure excedances $i$, and for each of them we have $\pi^{-1}(i)=\sigma^{-1}(i)$. Let $j$ be the greatest integer such that $\pi_{j} \neq \sigma_{j}$ (without loss of generality, we assume $\pi_{j}<\sigma_{j}$ ). In this case, $j$ is not a pure excedance for the two permutations. Thus, $\lambda(\pi)_{j}$ (resp. $\left.\lambda(\sigma)_{j}\right)$ is the sum of the label of $\left(j, \pi_{j}\right)$ (resp. $\left(j, \sigma_{j}\right)$ ) with the number of pure excedances 
$k<j$ such that $\pi^{-1}(k)<j$ (resp. $\sigma^{-1}(k)<j$ ). Since we have $\pi_{j}<\sigma_{j}$, the label of $\left(j, \pi_{j}\right)$ is less than the label of $\left(j, \sigma_{j}\right)$, and the number of pure excedances $k<j$ such that $\pi^{-1}(k)<j$ is less than or equal to the number of pure excedances $k<j$ such that $\sigma^{-1}(k)<j$. Then we have $\lambda(\pi)_{j}<\lambda(\sigma)_{j}$. Then $\lambda$ is an injective map, and thus a bijection.

Theorem 3.2. There is a one-to-one correspondence $\psi$ on the set $D_{n}$ of $n$-length derangements such that for any $\pi \in D_{n}$,

$$
\operatorname{pex} \pi=\operatorname{cyc} \psi(\pi) \text {. }
$$

Proof. Considering Theorem 2.3 and Theorem 3.1, we define for any $\pi \in D_{n}, \psi(\pi)=\phi(\sigma)$ where $\sigma$ is the permutation having $\lambda(\pi)$ as transposition array.

Theorem 3.3. The two bistatistics (pex, fix) and (pcyc, fix) are equidistribiuted on $S_{n}$.

Proof. Considering Theorem 3.2, we define the map $\bar{\psi}$ on $S_{n}$. Let $\pi^{\prime}$ be the permutation obtained from $\pi$ by deleting all fixed points and after rescaling as a permutation. Let $I=\left\{i_{1}, i_{2}, \ldots, i_{k}\right\}$ be the set of fixed points of $\pi$. Then, we set $\pi^{\prime \prime}=\psi\left(\pi^{\prime}\right)$. So, $\sigma=\bar{\psi}(\pi)$ is obtained from $\pi^{\prime \prime}$ by inserting fixed points $i \in I$ after a shift of all other entries in order to produce a permutation in $S_{n}$. By construction, we have pex $\pi=$ pcyc $\sigma$ and fix $\pi=$ fix $\sigma$ which completes the proof.

A byproduct of this theorem is

Corollary 3.1. The statistics cyc and pex + fix are equidistributed on $S_{n}$.

Also, a direct consequence of Theorems 2.3 and 3.3 is

Theorem 3.4. The two statistics pex and $\mathbf{d e s}_{2}$ are equidistributed on $S_{n}$.

Notice that Foata's first transformation is not a candidate for proving the equidistribution of pex and $\mathbf{d e s}_{2}$, while it transports exc to des. Combining Theorem 3.4 and Corollary 2.1 we have the following.

Corollary 3.2. For $0 \leq i \leq 2$, the patterns $p_{i}$ and pex are equipopular on $S_{n}$ (see Sequence A001705 in [15]).

Finally, we present two conjectures for future works.

Conjecture 3.1. The two bistatistics $\left(\mathbf{d e s}_{2}, \mathbf{c y c}\right)$ and (pex, cyc) are equidistributed on $S_{n}$.

Conjecture 3.2. The two bistatistics $\left(\mathbf{d e s}_{2}\right.$, des) and (pex, exc) are equidistributed on $S_{n}$.

It is interesting to remark that $(\mathbf{d e s}, \mathbf{c y c})$ and $(\mathbf{e x c}, \mathbf{c y c})$ are not equidistributed. Indeed, there are 3 permutations in $S_{3}$ having $\mathbf{e x c}=1$ and $\mathbf{c y c}=2$, namely $132,213,321$, but only 2 permutations with des $=1$ and $\mathbf{c y c}=2$, videlicet 132 and 213. So, if the Conjectures 3.1 and 3.2 are true then their proofs are probably independent.

\section{Acknowledgements}

We would like to greatly thank Vincent Vajnovszki for having offered us Conjecture 3.2 and the anonymous referees for their helpful comments and suggestions.

\section{References}

[1] J.-L. Baril, Statistics-preserving bijections between classical and cycle permutations, Inform. Process. Lett. 113 (2013), 17-22.

[2] J.-L. Baril and S. Kirgizov, The pure descent statistic on permutations, Discrete Math. $340: 10$ (2017), 2250-2558.

[3] J.-L. Baril and V. Vajnovszki, Popularity of patterns over d-equivalence classes of words and permutations, Theoret. Comput. Sci. 814 (2020), 249-258.

[4] F. Bergeron, N. Bergeron, R. B. Howlett and D. E. Taylor, A decomposition of the descent algebra of a finite Coxeter group, J. Algebraic Combin. 1 (1992), 23-44.

[5] M. Bóna, Surprising symmetries in objects counted by Catalan numbers, Electron. J. Combin. 19:1 (2012), Article P62. 
[6] P. Brändén and A. Claesson, Mesh patterns and the expansion of permutation statistics as sums of permutation patterns, Electron. J. Combin. 18:2 (2011), Article P5.

[7] S. Elizalde, Descent sets of cyclic permutations, Adv. in Appl. Math. 47.4 (2011), 688-709.

[8] S. Elizalde and J. M. Troyka, The number of cycles with a given descent set, Sém. Lothar. Combin. 80 (2018) Article \#8.

[9] D. Foata and M. P. Schützenberger, Théorie Géométrique des Polynômes Euleriens, Lecture Notes in Math. 138, Springer-Verlag, Berlin, 1970.

[10] D. Foata and G.-N. Han, Decreases and descents in words, Sém. Lothar. Combin. 58 (2007), Article B58a.

[11] A. Garsia and C. Reutenauer, A decomposition of Solomon's descent algebra, Adv. Math. 77 (1989), $189-262$.

[12] I. Gessel and G. Viennot, Binomial determinants, paths, and hook length formulae, Adv. Math. 58 (1985), 300-321.

[13] S. Kitaev and P.B. Zhang, Distributions of mesh patterns of short lengths, Adv. in Appl. Math. 110 (2019), 1-32.

[14] P.A. MacMahon, Combinatory Analysis, Volumes 1 and 2, Cambridge Univ. Press, Cambridge, UK, 1915 (reprinted by Chelsea, New York, 1955).

[15] N.J.A. Sloane, The On-line Encyclopedia of Integer Sequences, available electronically at http://oeis.org.

[16] R.P. Stanley, Enumerating Combinatorics, Volume 2, Cambridge University Press, 1999.

[17] R.M. Wilson and J.H. van Lint, A course in combinatorics, Volume I, Cambridge University Press, 2002. 